\title{
Impactos da exótica invasora Boerhavia diffusa L. sobre a diversidade de espécies do estrato herbáceo e arbustivo autóctone de uma área ripária na Caatinga, Sergipe, Brasil
}

Impacts of the exotic invasive Boerhavia diffusa L. on the diversity of native herbaceous and shrub strata of riparian area in Caatinga, Sergipe, Brazil

\author{
L. A. Santos ${ }^{1 *} ;$ J. R. Fabricante ${ }^{1}$ \\ ${ }^{1}$ Laboratório de Ecologia e Conservação da Biodiversidade, Departamento de Biociências, Universidade Federal de \\ Sergipe, CEP: 49500-000, Itabaiana - SE, Brasil. \\ *lizandraalmeidasantos32@hotmail.com \\ (Recebido em 17 de setembro de 2018; aceito em 21 de dezembro de 2018)
}

\begin{abstract}
Nosso objetivo foi estudar os impactos causados pela espécie exótica invasora Boerhavia diffusa sobre o estrato herbáceo e arbustivo em uma área ripária de Caatinga em Canindé de São Francisco, SE. Para isso, utilizamos o método de parcelas e amostramos dois ambientes nos quais todas as espécies herbáceas foram contabilizadas e estimados os parâmetros estruturais das comunidades. Amostramos 13 espécies e 513 indivíduos no ambiente invadido e 36 espécies e 1.056 indivíduos no ambiente não invadido. A diversidade de espécies baseada no índice de Shannon-Wiener (H') foi de 1,69 para o ambiente invadido e de 2,63 para o ambiente não invadido. Nossos resultados sugerem que Boerhavia diffusa afeta de maneira negativa a diversidade de espécies do estrato herbáceo e arbustivo autóctone da área estudada.

Palavras-chave: Invasão biológica; Savana Estépica; Impacto ambiental.
\end{abstract}

Our objective was to study the impacts caused by invasive species Boerhavia diffusa on the herbaceous and shrub stratum in a riparian area of Caatinga in Canindé de São Francisco, SE. For this, we used the plots method and sampled two environments in which all the herbaceous species were counted and estimated the structural parameters of the communities. We sampled 13 species and 513 individuals in the invaded environment and 36 species and 1,056 individuals in the not invaded. The Shannon-Wiener species diversity was 1.69 for the invaded environment and 2.63 for the environment not invaded. Our results suggest that Boerhavia diffusa affects negatively the species diversity of autochthonous herbaceous and shrub stratum of the study area.

Keywords: Biological invasion; Dry Forest; Environment impact.

\section{INTRODUÇÃO}

A flora da Caatinga é composta por 4.478 espécies [1] e se caracteriza por apresentar uma alta variedade fisionômica e uma grande diversidade de espécies endêmicas [2]. Apesar desta alta diversidade de espécies, as fitofisionomias da Caatinga encontram-se fortemente degradadas [3, 4]. Esta degradação tem promovido a diminuição da riqueza e diversidade de espécies nativas, além de ocasionar a redução das funções ecológicas, podendo atuar como um facilitador para o processo de invasão biológica $[5,6,7]$.

Invasão biológica é o processo de introdução e consequente adaptação de espécies que não fazem parte naturalmente de um determinado ecossistema, e que passam a ser agentes de mudanças e ameaça a diversidade nativa $[7,8,9]$, podendo levar à extinção de espécies, seja de forma direta pela competição por recursos, ou de forma indireta através de alterações dos processos ecossistêmicos locais $[10,11]$.

Devido à ausência de inimigos naturais, tais como patógenos, predadores ou competidores na região de introdução, as espécies exóticas invasoras podem apresentar altas taxas de crescimento populacional, bem acima do que ocorre em sua região original [12]. Em plantas, uma das características que favorecem a capacidade de invasão de uma espécie está relacionado diretamente com sua alta capacidade de dispersão, reproduzir, crescer rapidamente desde a sua 
germinação até o estágio reprodutivo, bem como sua plasticidade fenotípica que lhe permitirá adaptar-se ao estresse ambiental do novo ambiente [13].

Diante do exposto, nos domínios da Caatinga são reconhecidas 205 espécies de plantas exóticas [14], das quais algumas com comprovado impacto sobre a biota [15, 16, 17,18, 19, 20]. Uma das espécies que vem demostrando comportamento invasor na Caatinga é a Boerhavia diffusa L. Conhecida popularmente como pega-pinto, corresponde a uma herbácea pertencente à família Nyctaginaceae, originária da Índia [21], que diminui significativamente a germinação e o desenvolvimento de outras espécies por meio de inibição com aleloquímicos [22], provocando prejuízos importantes para agricultura, pecuária e diversos setores de interesse humano [23]. Já sobre a biodiversidade autóctone, não existem informações sobre os impactos dessa exótica invasora. Dessa forma, o presente trabalho teve como objetivo avaliar os efeitos de $B$. diffusa sobre a composição, riqueza e diversidade de plantas nativas de uma área ripária de Caatinga.

\section{MATERIAL E MÉTODOS}

O estudo foi realizado em uma área ripária de Caatinga a jusante da barragem de Xingó, Município de Canindé de São Francisco, SE (9`38’23,33” S; 3746’46,03” W - altitude média de $17 \mathrm{~m})$. O sítio estudado tem por volta de 40 ha e apresenta vários indícios de degradação, a exemplo de pontos de depósito de lixo, esgoto, presença de animais de pastoreio, características essas que tornam determinados ecossistemas mais suscetíveis as invasões biológicas. O clima da região é o semiárido quente (BSh de acordo com a classificação de Köppen-Geiger) com precipitação média anual de $500 \mathrm{~mm}$ e temperatura média de $24^{\circ} \mathrm{C}$ a $26^{\circ} \mathrm{C}$ [24]. Os solos predominantes são do tipo Neossolos, Luvissolos e Planossolos [25].

Para a avaliação dos impactos causados por $B$. diffusa sobre a vegetação autóctone da Caatinga foram estabelecidas 20 parcelas de $1 \times 1 \mathrm{~m}$, das quais 10 unidades amostrais no sítio invadido pela espécie (I) e outras 10 no sítio não invadidos (NI) com as mesmas características biofísicas (mesmo ambiente) e o mais próximo possível das anteriores (sítios invadidos). No interior dessas unidades amostrais foram contabilizados todos os indivíduos de todas as espécies presentes. Ainda, cada parcela foi subdividida em 100 subparcelas de $10 \times 10 \mathrm{~cm}$, onde computamos o número de subparcelas preenchidas por cada espécie.

A suficiência amostral foi avaliada para cada ambiente (I e NI) por meio da curva de acumulação de espécies. O número de espécies e indivíduos foram previamente submetidos aos testes de normalidade dos resíduos e homocedasticidade das variâncias e os valores medianos foram comparados entre os ambientes invadido (I) e não invadido (NI) através do teste de MannWhitney (U) [26] ( $\mathrm{p}<0,05)$. A diversidade de cada sítio foi obtida por meio de Shannon-Wiener (H') [27] e a equabilidade pelo índice de Pielou (J') [28]. Verificamos diferenças entre as diversidades pelo teste $\mathrm{t}[29](\mathrm{p} \leq 0,05)$.

Para avaliar a similaridade florística entre os sítios invadidos e não invadidos utilizou-se o coeficiente de Jaccard $(\mathrm{Sj})$ [30] e a dissimilaridade foi obtida por Bray-Curtis [31]. O grau de ajuste dos agrupamentos formados nas análises foi verificado pelo coeficiente de correlação cofenética [32]. Para avaliar se existiam diferenças significativas entre as unidades amostrais foram realizados testes de permutação ANOSIM (oneway) [33]. Para a visualização gráfica dos resultados foram feitas análises de NMDS (Escalonamento Multidimensional Não Métrico).

Para cada espécie foram calculados os parâmetros fitossociológicos [30,34]: densidade absoluta (DA) e relativa (DR), frequência absoluta (FA) e relativa (FR), cobertura absoluta (CA) e relativa (CR) e valor de importância (VI). Foi calculado também o índice de impacto ambiental de espécies exóticas (IIAE), a partir do coeficiente do impacto ambiental. De acordo com Reaser et al. (2007) [35]: varia de -1 a 1 e quanto mais próximo do extremo negativo, mais acentuado é o grau da invasão/impacto.

Todas as espécies amostradas foram coletadas, herborizadas e depositadas no Herbário ASE, Universidade Federal de Sergipe, São Cristóvão, SE. Elaboramos a classificação taxonômica de acordo com o Sistema APG IV (2016) [36] e a grafia dos nomes dos autores das espécies segundo a Lista de Espécies da Flora do Brasil [37]. As análises foram realizadas por meio dos softwares MVSP 3.1 [38] e Past 2.17 [39]. 


\section{RESULTADOS E DISCUSSÃO}

Amostramos 12 espécies (uma morfoespécie), distribuídas em 11 gêneros e 11 famílias no ambiente invadido (I). Já no ambiente não invadido (NI), encontramos 36 espécies (uma morfoespécie), 32 gêneros e 18 famílias (Tabela 1).

Tabela 1. Espécies herbáceas e arbustivas amostradas em uma área ripária de Caatinga a jusante da barragem de Xingó, Canindé de São Francisco, SE. I = ambiente invadido; NI = ambiente não invadido; 1 = presença da espécie; $0=$ ausência da espécie.

\begin{tabular}{|c|c|c|c|c|}
\hline Família & Espécie & $\mathrm{I}$ & NI & Tombo \\
\hline \multirow[t]{3}{*}{ Asteraceae } & Blainvillea acmella (L.) Philipson & 0 & 1 & 38822 \\
\hline & Delilia biflora (L.) Kuntze. & 0 & 1 & 38836 \\
\hline & Pectis sp. & 0 & 1 & - \\
\hline Amarathaceae & Alternanthera tenella Colla & 0 & 1 & 38832 \\
\hline Cactaceae & Tacinga inamoena (K.Schum.) N.P.Taylor \& Stuppy & 0 & 1 & - \\
\hline \multirow[t]{2}{*}{ Commelinaceae } & Commelina benghalensis $\mathrm{L}$. & 0 & 1 & 38825 \\
\hline & Commelina diffusa Burm.f. & 0 & 1 & 38823 \\
\hline \multirow[t]{6}{*}{ Euphorbiaceae } & Acalypha poiretii Spreng. & 0 & 1 & 38833 \\
\hline & Astraea lobata (L.) Klotzsch & 0 & 1 & 38830 \\
\hline & Croton hirtus L'Hér. & 0 & 1 & 38838 \\
\hline & Cnidoscolus urens (L.) Arthur & 0 & 1 & 38829 \\
\hline & Euphorbia hyssopifolia $\mathrm{L}$. & 1 & 1 & - \\
\hline & Phyllanthus niruri L. & 0 & 1 & - \\
\hline \multirow[t]{3}{*}{ Fabaceae } & Senna alata (L.) Roxb. & 0 & 1 & 38824 \\
\hline & Tephrosia sp. & 1 & 1 & 38817 \\
\hline & Macroptilium lathyroides (L.) Urb. & 0 & 1 & - \\
\hline Lamiaceae & Mesosphaerum suaveolens (L.) Kuntze & 1 & 1 & 38821 \\
\hline Loasaceae & Mentzelia aspera $\mathrm{L}$. & 0 & 1 & 38841 \\
\hline Loganiaceae & Spigelia anthelmia $\mathrm{L}$ & 0 & 1 & 38826 \\
\hline \multirow[t]{2}{*}{ Malvaceae } & Herissantia crispa (L.) Brizicky & 1 & 1 & 38812 \\
\hline & Melochia tomentosa $\mathrm{L}$. & 0 & 1 & 38828 \\
\hline Molluginaceae & Mollugo verticillata $\mathrm{L}$. & 1 & 1 & 38815 \\
\hline Nyctaginaceae & Boerhavia diffusa $\mathrm{L}$. & 1 & 0 & - \\
\hline \multirow[t]{2}{*}{ Oxalidaceae } & Oxalis divaricata Mart. ex Zucc. & 0 & 1 & 38834 \\
\hline & Oxalis glaucescens Norlind & 0 & 1 & 38835 \\
\hline \multirow[t]{3}{*}{ Poaceae } & Digitaria sp. & 0 & 1 & 38831 \\
\hline & Dactyloctenium aegyptium (L.) Willd & 1 & 0 & 38814 \\
\hline & Eragrostis tenella (L.) P.Beauv. ex Roem. \& Schult. & 0 & 1 & 38837 \\
\hline
\end{tabular}




$\begin{array}{lllcc}\text { Portulacaceae } & \text { Portulaca elatior Mart. } & 1 & 1 & 38811 \\ & \text { Portulaca oleracea } \text { L., } & 0 & 1 & - \\ \text { Rubiaceae } & \text { Borreria scabiosoides Cham. \& Schltdl. } & 0 & 1 & - \\ & \text { Hexasepalum teres (Walter) J. H. Kirkbr. } & 0 & 1 & 38839 \\ & \text { Richardia grandiflora (Cham. \& Schltdl.) Steud. } & 1 & 1 & 38816 \\ \text { Solanaceae } & \text { Physalis angulata } \text { L. } & 1 & 1 & 38818 \\ \text { Turneraceae } & \text { Turnera subulata } \text { Sm. } & 0 & 1 & 38827 \\ \text { Zygophyllaceae } & \text { Kallstroemia tribuloides (Mart.) Steud. } & 1 & 1 & 38820 \\ \text { Família ident. } & \text { Morfoespécie 1 } & 1 & 1 & - \\ & \text { Morfoespécie 2 } & 0 & 1 & -\end{array}$

Os resultados da curva de acumulação de espécies indicam uma tendência de estabilização no número de táxons nas parcelas (Figura 1). Esse resultado sugere que o esforço amostral utilizado foi suficiente para amostrar satisfatoriamente os ambientes estudados.

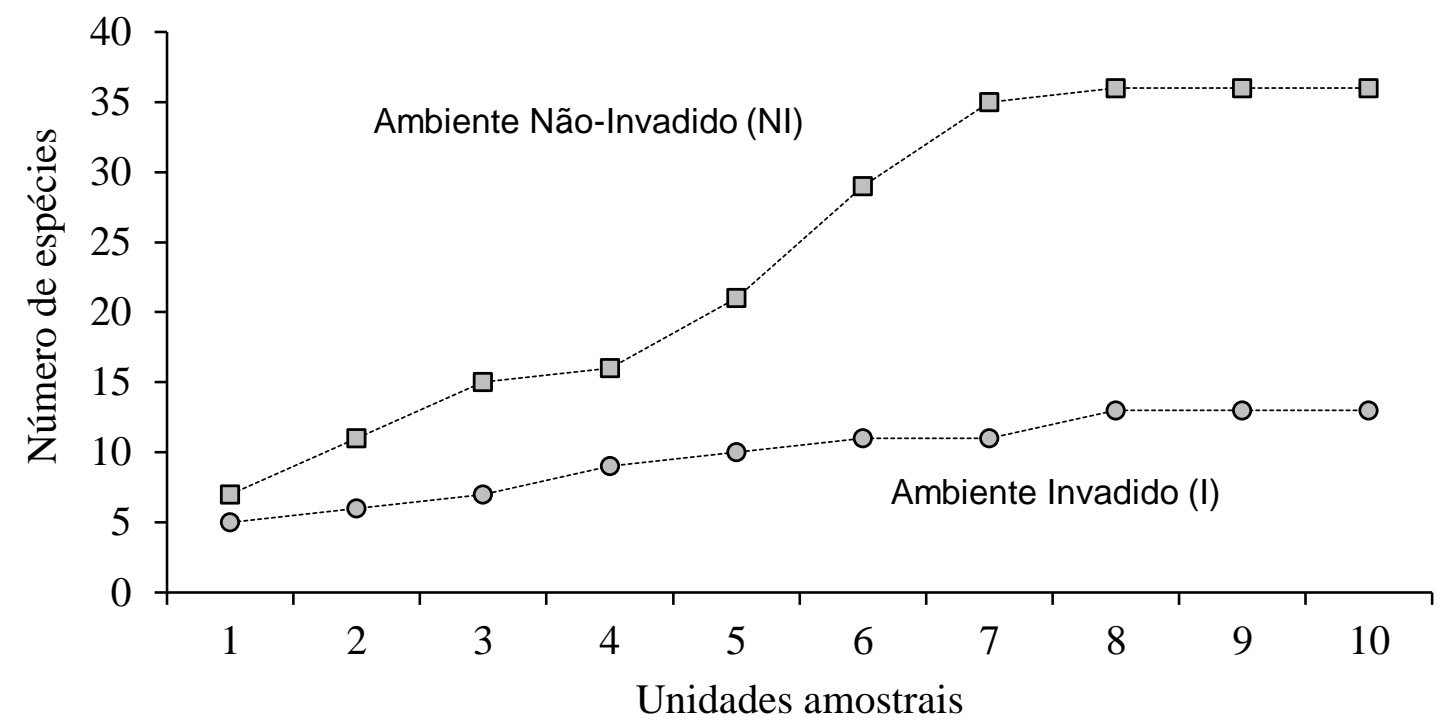

Figura 1. Curva de acumulação de espécies do estrato herbáceo e arbustivo para os ambientes estudados em uma área riparia de Caatinga a jusante da barragem de Xingó, Canindé de São Francisco, SE.

No total foram amostrados 513 indivíduos no ambiente invadido, dos quais, 241 da exótica invasora estudada. Já no ambiente não invadido foram amostrados 1.056 indivíduos. De acordo com o teste de Mann-Whitney [26], o valor mediano de espécies $(Z=7,50 ; Z(U)=3,21 ; p<0,01)$ e indivíduos $(Z=6,50 ; Z(U)=3,29 ; p<0,01)$ foi significativamente maior no ambiente não invadido (NI) quando comparado ao ambiente invadido (I).

A diversidade de espécies obtida para o ambiente não invadido (NI) foi de $\mathrm{H}^{\prime}=2,63$ e para o invadido (I) foi de $\mathrm{H}^{\prime}=1,69$. Com diferença significativa entre os dois ambientes $(\mathrm{t}=15,42 ; \mathrm{p}<$ $0,01)$, apresentando maior diversidade no ambiente sem a presença da espécie $B$. diffusa. O valor de equabilidade foi maior no ambiente não invadidos $\left(\mathrm{J}^{\prime}=0,74\right)$ quando comparado com o do ambiente invadido $\left(\mathrm{J}^{\prime}=0,66\right)$. Este resultado evidencia a dominância da espécie exótica invasora no ambiente em relação às demais espécies, fazendo com que a equabilidade fosse sensivelmente diminuída, refletindo sobre a diversidade do ambiente invadido. 
Observa-se na análise de similaridade a formação de dois grandes grupos de parcelas: um formado pelas unidades amostrais do ambiente invadido (mais consistentes) e outro pelas do ambiente não invadido (Figura 2). O mesmo foi observado nos resultados da análise de dissimilaridade de Bray-Curtis (Figura 3).

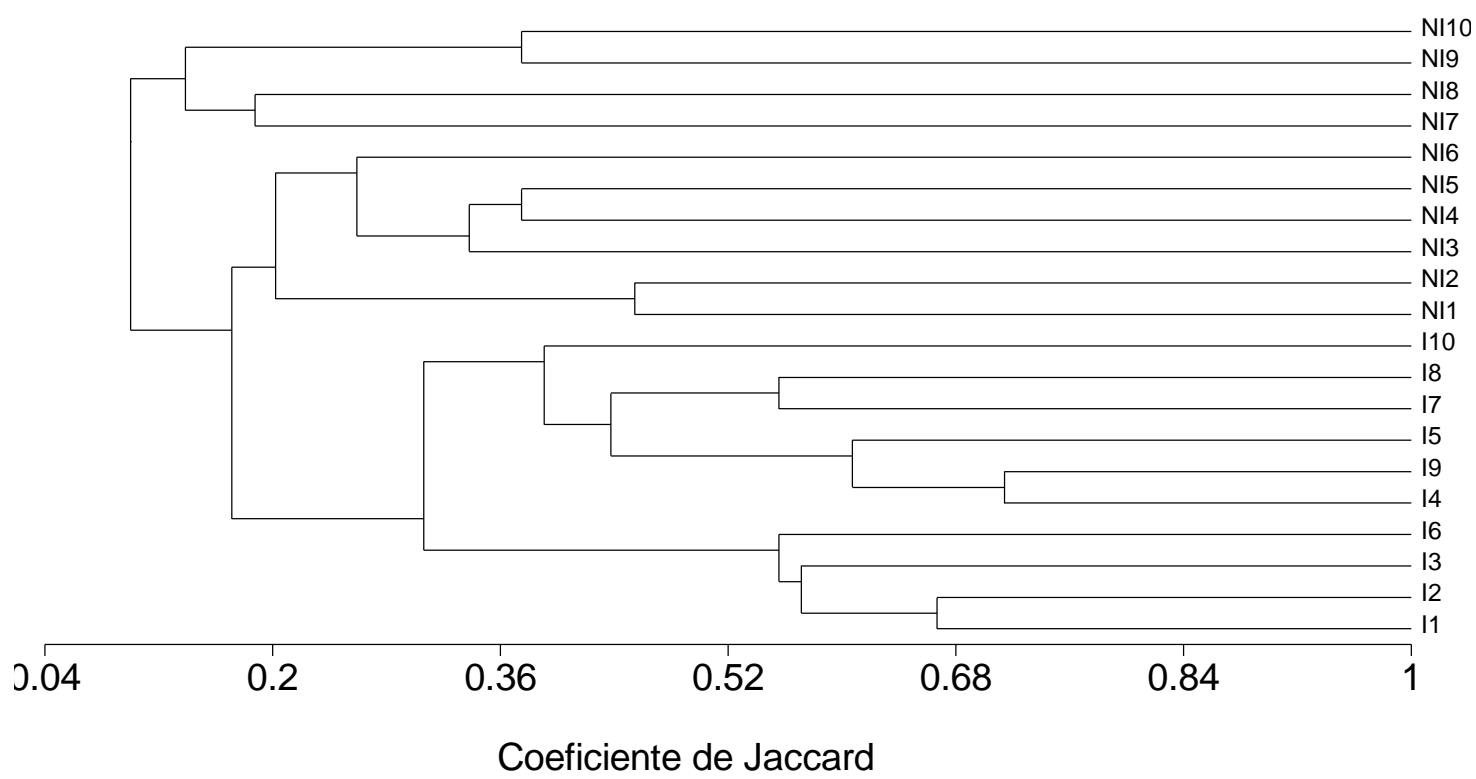

Figura 2. Análise de similaridade de Jaccard para as parcelas estudadas em uma área ripária de Caatinga a jusante da barragem de Xingó, Canindé de São Francisco, SE. I = parcelas do ambiente invadido; NI = parcelas do ambiente não invadido.

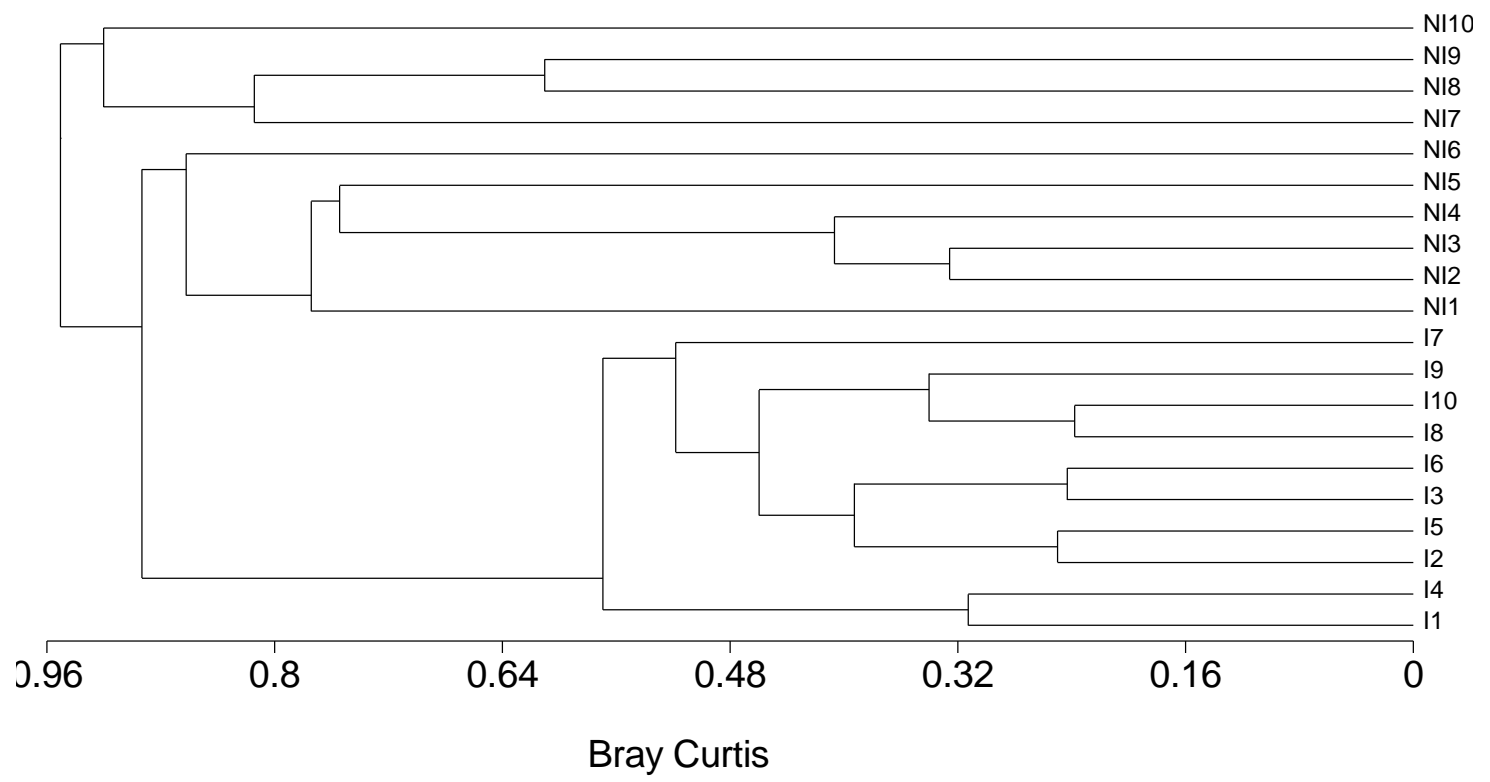

Figura 3. Resultado da análise de dissimilaridade de Bray-Curtis para as parcelas estudadas em uma área ripária de Caatinga a jusante da barragem de Xingó, Canindé de São Francisco, SE. Sendo: I = parcelas do ambiente invadido; $N I=$ parcelas do ambiente não invadido.

Foram encontradas diferenças significativas entre I e NI (ANOSIM), tanto por Jaccard ( $p \leq$ $0,01)$, como por Bray-Curtis ( $\mathrm{p} \leq 0,01)$. Isso indica que as réplicas (parcelas) de cada ambiente são mais similares (ou menos dissimilares) entre si, do que com as réplicas do outro ambiente e vice-versa. Através da análise gráfica de NMDS foi possível confirmar a formação dos dois 
grupos de parcelas relatados nas análises anteriores. Os resultados foram semelhantes quando utilizamos Jaccard (Figura 4) e Bray-Curtis (Figura 5).

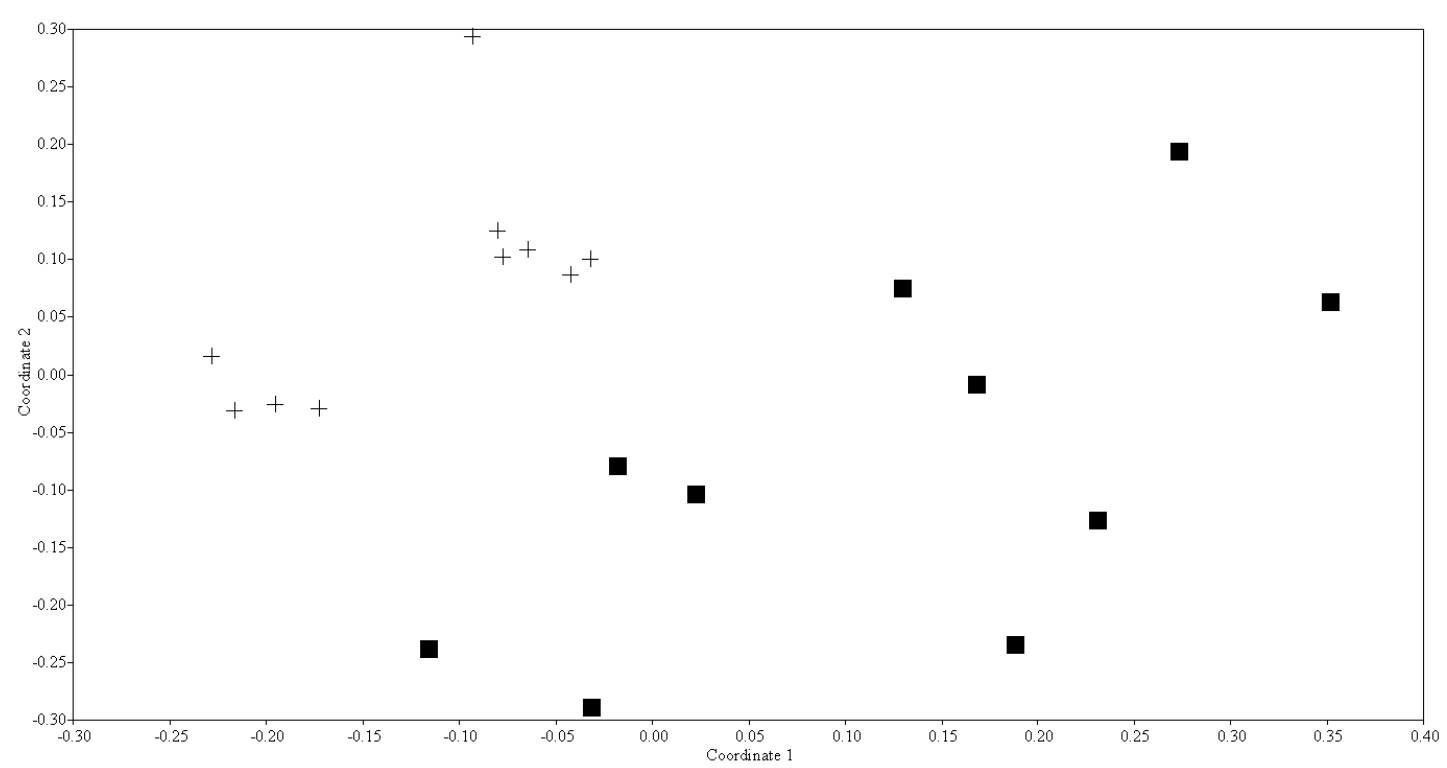

Figura 4. Análise de Escalonamento Multidimensional Não-Métrico (NMDS) por Jaccard para uma área ripária de Caatinga a jusante da barragem de Xingó, Canindé de São Francisco, SE. Cruzes = unidades amostrais do ambiente invadido por Boerhavia diffusa; Quadrados = unidades amostrais do ambiente não invadido. Stress $=0,23$.

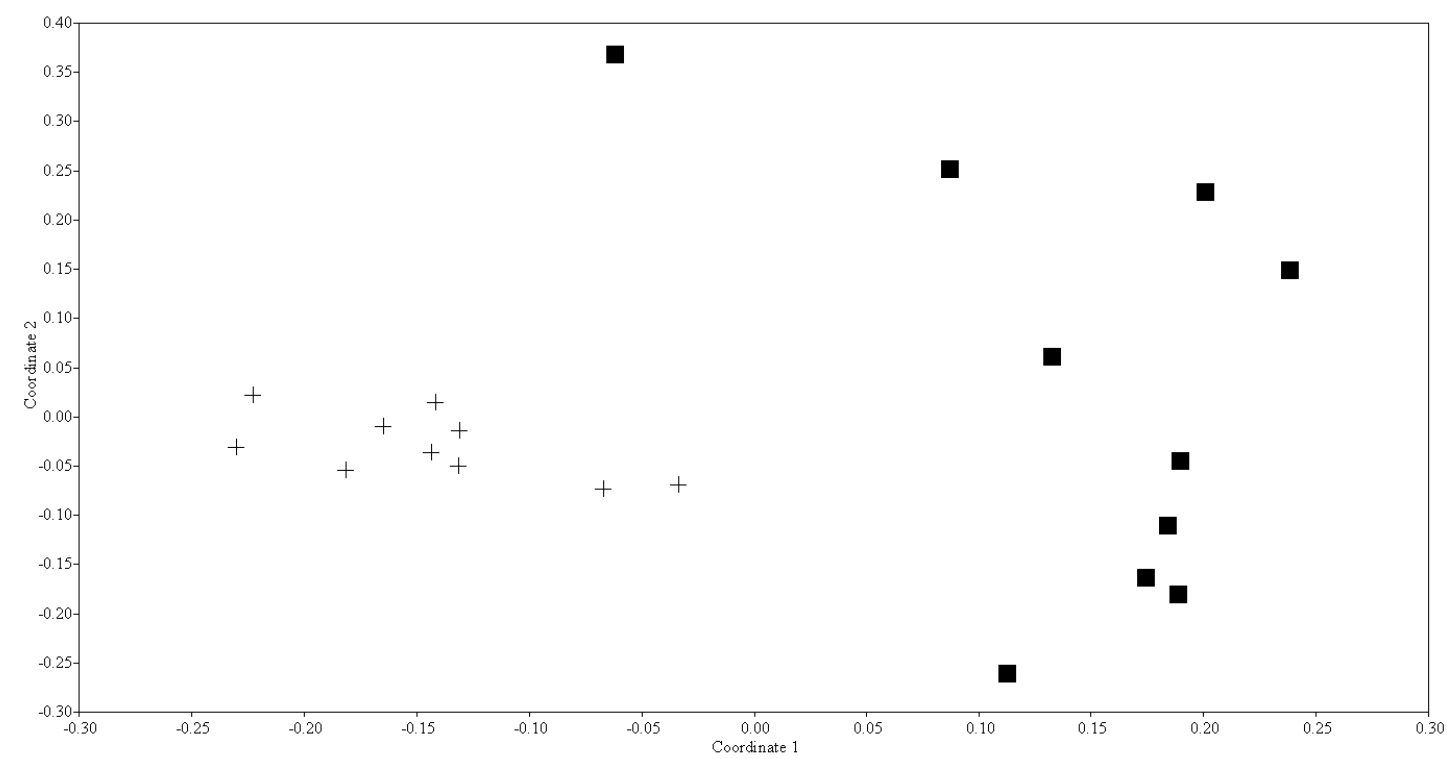

Figura 5. Análise de Escalonamento Multidimensional Não-Métrico (NMDS) por Bray-Curtis para uma área ripária de Caatinga a jusante da barragem de Xingó, Canindé de São Francisco, SE. Cruzes = unidades amostrais do ambiente invadido por Boerhavia diffusa; quadrados = unidades amostrais do ambiente não invadido. Stress $=0,2$.

Observou-se na estrutura da vegetação os efeitos da invasão da Boerhavia diffusa (Tabela 2). No ambiente invadido, a espécie exótica invasora foi a que apresentou o maior valor de importância (VI = 153,90; VI = 51,30\%), em razão de ter apresentado a maior densidade (DA = 24,10 indivíduos $\left.\mathrm{m}^{2}, \mathrm{DR}=46,98 \%\right)$, frequência $(\mathrm{FA}=100 \%$; $\mathrm{FR}=17,86 \%)$ e cobertura $(\mathrm{CA}=$ 75\%; $\mathrm{CR}=89 \%$ ). A segunda espécie com maior valor de importância, Kallstroemia tribuloides 
(Mart.) Steud (VI $=28,24)$, apresentou uma densidade absoluta de apenas 7,5 indivíduos $\mathrm{m}^{2}$ (DR $=14,62 \%)$, uma frequência absoluta de $60 \%(\mathrm{FR}=10,7 \%)$ e uma cobertura absoluta de $2,45 \%$ $(\mathrm{CR}=2,91 \%)$. 


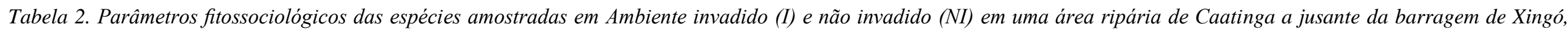

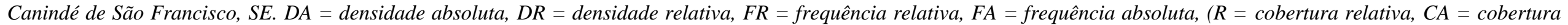
absoluta, $V I=$ valor de importância .

\begin{tabular}{|c|c|c|c|c|c|c|c|c|c|c|c|c|c|c|c|c|}
\hline \multirow[t]{2}{*}{ Espécies } & \multicolumn{8}{|c|}{ Ambiente Invadido } & \multicolumn{8}{|c|}{ Ambiente Não Invadido } \\
\hline & DA & DR & FA & FR & $\mathrm{CA}$ & $\mathrm{CR}$ & VI & $\% \mathrm{VI}$ & DA & DR & FA & FR & $\mathrm{CA}$ & $\mathrm{CR}$ & VI & $\% \mathrm{VI}$ \\
\hline Boerhavia diffusa & 24,1 & 46,98 & 100 & 17,86 & 75 & 89,07 & 153,9 & 51,3 & - & - & - & - & - & - & - & - \\
\hline Kallstroemia tribuloides & 7,5 & 14,62 & 60 & 10,71 & 2,45 & 2,91 & 28,24 & 9,415 & 7,3 & 6,913 & 60 & 6,593 & 7,65 & 11,18 & 24,68 & 8,227 \\
\hline Richardia grandiflora & 6,4 & 12,48 & 70 & 12,5 & 1,85 & 2,197 & 27,17 & 9,058 & 32,7 & 30,97 & 50 & 5,495 & 23,2 & 33,89 & 70,35 & 23,45 \\
\hline Mesosphaerum suaveolens & 5,3 & 10,33 & 70 & 12,5 & 1,8 & 2,138 & 24,97 & 8,323 & 2,7 & 2,557 & 50 & 5,495 & 1,1 & 1,607 & 9,658 & 3,219 \\
\hline Mollugo verticillata. & 2,5 & 4,873 & 50 & 8,929 & 0,15 & 0,178 & 13,98 & 4,66 & 5,2 & 4,924 & 60 & 6,593 & 1,8 & 2,63 & 14,15 & 4,716 \\
\hline Portulaca elatior & 1,9 & 3,704 & 50 & 8,929 & 0,65 & 0,772 & 13,4 & 4,468 & 0,4 & 0,379 & 20 & 2,198 & 0,45 & 0,657 & 3,234 & 1,078 \\
\hline Dactyloctenium aegyptium & 0,8 & 1,559 & 50 & 8,929 & 0,7 & 0,831 & 11,32 & 3,773 & - & - & - & - & - & - & - & - \\
\hline Physalis angulata & 1,4 & 2,729 & 40 & 7,143 & 0,35 & 0,416 & 10,29 & 3,429 & 0,3 & 0,284 & 20 & 2,198 & 0,6 & 0,877 & 3,358 & 1,119 \\
\hline Tephrosia sp. & 0,9 & 1,754 & 30 & 5,357 & 0,5 & 0,594 & 7,705 & 2,568 & 7,2 & 6,818 & 50 & 5,495 & 4,85 & 7,085 & 19,4 & 6,466 \\
\hline Euphorbia hyssopifolia & 0,2 & 0,39 & 10 & 1,786 & 0,05 & 0,059 & 2,235 & 0,745 & 0,4 & 0,379 & 10 & 1,099 & 0,15 & 0,219 & 1,697 & 0,566 \\
\hline Morfoespécie 1 & 0,1 & 0,195 & 10 & 1,786 & 0,1 & 0,119 & 2,09 & 0,7 & - & - & - & - & - & - & - & - \\
\hline Herissantia crispa & 0,1 & 0,195 & 10 & 1,786 & 0,05 & 0,059 & 2,04 & 0,68 & 0,7 & 0,663 & 20 & 2,198 & 0,35 & 0,511 & 3,372 & 1,124 \\
\hline Acalypha poiretii & - & - & - & - & - & - & - & - & 7,7 & 7,292 & 50 & 5,495 & 2,95 & 4,31 & 17,1 & 5,699 \\
\hline Astraea lobata & & & & & & & & & 0,4 & 0,379 & 20 & 2,198 & 0,2 & 0,292 & 2,869 & 0,956 \\
\hline Mentzelia aspera & & & & & & & & & 4,8 & 4,545 & 40 & 4,396 & 3,9 & 5,698 & 14,64 & 4,88 \\
\hline Commelina benghalensis & & & & & & & & & 0,7 & 0,663 & 20 & 2,198 & 0,35 & 0,511 & 3,372 & 1,124 \\
\hline Alternanthera tenella & & & & & & & & & 1,1 & 1,042 & 40 & 4,396 & 0,3 & 0,438 & 5,876 & 1,959 \\
\hline Digitaria sp. & & & & & & & & & 5,3 & 5,019 & 60 & 6,593 & 3,5 & 5,113 & 16,73 & 5,575 \\
\hline Hexasepalum teres & - & - & - & - & - & - & - & - & 10,1 & 9,564 & 50 & 5,495 & 4,75 & 6,939 & 22 & 7,333 \\
\hline
\end{tabular}


Turnera subulata

Oxalis divaricata

Croton hirtus

Eragrostis tenella

Senna alata

Oxalis glaucescens

Spigelia anthelmia

Delilia biflora

Melochia tomentosa

Blainvillea acmella

Portulaca oleracea

Commelina diffusa

Pectis sp.

Tacinga inamoena

Cnidoscolus urens

Borreria scabiosoides

Morfoespécie 2

Macroptilium lathyroides

Phyllanthus niruri

Total

51,2

$\begin{array}{cccccccc}0,1 & 0,095 & 10 & 1,099 & 0,4 & 0,584 & 1,778 & 0,593 \\ 0,1 & 0,095 & 10 & 1,099 & 0,05 & 0,073 & 1,267 & 0,422 \\ 0,3 & 0,284 & 10 & 1,099 & 0,15 & 0,219 & 1,602 & 0,534 \\ 0,7 & 0,663 & 20 & 2,198 & 0,2 & 0,292 & 3,153 & 1,051 \\ 2,1 & 1,989 & 10 & 1,099 & 1,2 & 1,753 & 4,841 & 1,614 \\ 0,9 & 0,852 & 10 & 1,099 & 0,95 & 1,388 & 3,339 & 1,113 \\ 2 & 1,894 & 40 & 4,396 & 0,7 & 1,023 & 7,312 & 2,437 \\ 1 & 0,947 & 10 & 1,099 & 0,8 & 1,169 & 3,215 & 1,072 \\ 0,3 & 0,284 & 30 & 3,297 & 0,1 & 0,146 & 3,727 & 1,242 \\ 0,2 & 0,189 & 10 & 1,099 & 0,05 & 0,073 & 1,361 & 0,454 \\ 1,7 & 1,61 & 30 & 3,297 & 0,2 & 0,292 & 5,199 & 1,733 \\ 1,8 & 1,705 & 10 & 1,099 & 1 & 1,461 & 4,264 & 1,421 \\ 0,9 & 0,852 & 10 & 1,099 & 0,4 & 0,584 & 2,536 & 0,845 \\ 0,1 & 0,095 & 10 & 1,099 & 0,1 & 0,146 & 1,34 & 0,447 \\ 0,2 & 0,189 & 10 & 1,099 & 0,1 & 0,146 & 1,434 & 0,478 \\ 3,3 & 3,125 & 10 & 1,099 & 2,4 & 3,506 & 7,73 & 2,577 \\ 0,9 & 0,852 & 20 & 2,198 & 0,85 & 1,242 & 4,292 & 1,431 \\ 0,3 & 0,284 & 10 & 1,099 & 0,15 & 0,219 & 1,602 & 0,534 \\ 1,3 & 1,231 & 10 & 1,099 & 0,55 & 0,804 & 3,133 & 1,044 \\ 1.056 & 100 & 910 & 100 & 66,65 & 100 & 300 & 100 \\ & & & & & & & \end{array}$


Comparativamente no ambiente não invadido, sete espécies juntas obtiveram o mesmo valor de importância que a exótica invasora apresentou no ambiente invadido. Richardia grandiflora foi a espécie com o maior valor de importância ( $\mathrm{VI}=70,35)$, seguida por Kallstroemia tribuloides (Mart.) Steud. (VI $=24,68 \%)$, Acalypha poiretii Spreng (VI $=17,1 \%)$, Digitaria sp. (VI = $16,73 \%)$, Mollugo verticillata $\mathrm{L}$. $(\mathrm{VI}=14,15 \%)$, Mesosphaerum suaveolens $(\mathrm{L}$.$) Kuntze (VI =$ $9,6 \%$ ) e Tacinga inamoena (K.Schum.) N.P.Taylor \& Stuppy (VI = 1,3\%).

O valor do índice de impacto ambiental (IIAE) foi de -0,0267. Esse resultado confirma os efeitos negativos da espécie exótica invasora sobre a flora nativa apresentados até aqui. Dentre os principais impactos provocados por espécies exóticas invasoras nos ecossistemas invadidos, destacam-se a diminuição da riqueza e da diversidade de espécies [40]. Nossos resultados corroboram com essa afirmativa acima. Ainda, esses resultados são similares aos encontrados em estudos realizados em outras áreas. O estudo realizado em um fragmento de Mata Atlântica invadida por Artocarpus heterophyllus Lam. [50], os trabalhos realizados em áreas da Caatinga invadidas por Nicotiana glauca Graham [19], Prosopis juliflora (Sw) DC. [17, 51, 16, 52] e por Calotropis procera (Aiton) W.T. Aiton [53], e em uma área de Cerrado invadida por Melinis minutiflora P. Beauv [9] apresentaram resultados semelhantes ao do presente trabalho. Tais achados sugerem que independentemente da espécie exótica estudada e do bioma considerado, os efeitos das exóticas invasoras sobre a biota tendem a ser muito parecidos.

Tais resultados demonstram que os impactos ocasionados pela $B$. diffusa são mais evidenciados quando se leva em consideração não apenas a presença e ausência das espécies, mas também a abundância das mesmas. Dentre outros impactos ocasionados pelas espécies exóticas invasoras destacamos as mudanças na composição estrutural do ambiente [ver 41].

Quando comparado as características estruturais das comunidades invadidas por $B$. diffusa com todas as espécies presente no ambiente não-invadido, observamos que há poucas diferenças, visto que a população da exótica invasora se desenvolveu com características estruturais semelhantes às da comunidade nativa, substituindo-a em todas suas características. Sendo uma das principais consequências do processo de invasão biológica a exclusão de espécies, aumento na densidade por área e diminuição do número de indivíduos das espécies nativas [42, 43, 44, 45, $46,47,17,48,16,49]$.

É importante destacar que várias espécies nativas importantes para o funcionamento dos ecossistemas regionais não estavam presentes nos sítios invadidos. Segue alguns exemplos dessas espécies: Cnidoscolus urens (L.) Arthur, Melochia tomentosa L. Senna alata (L.) Roxb, Tacinga inamoena (K.Schum.) N.P.Taylor \& Stuppy, Turnera subulata sm. A espécie Cnidoscolus urens, pertencente à família Euphorbiaceae [54] possui uma ampla distribuição geográfica, ocorrendo desde a porção oriental do México até a Argentina [55]. No Brasil a espécie possui ocorrência no Nordeste, Sudeste e Centro-Oeste [56]. Com florescimento e frutificação no ano inteiro [57], sendo fonte de alimento para beija-flores [58] e abelhas [59].

Pertencente à família Fabaceae, Senna alata é uma planta perene arbustiva, que se propaga apenas por sementes. É uma espécie pantropical, podendo ser encontrada no Brasil desde o Norte até o Sudeste [60]. O táxon é fonte de alimento para abelhas, formigas e vespas [61]. Além disso a espécie pertence a um gênero muito importante de plantas fixadoras de nitrogênio e comumente utilizadas em projetos de recuperação de áreas degradadas [62].

Nativa do Brasil, Melochia tomentosa apresenta características que a torna promissora em projetos de recuperação de áreas degradadas [53]. Ela possui alto sucesso reprodutivo, mecanismos de adaptação a regiões com secas prolongadas e capacidade de colonizar habitats perturbados [63]. O táxon ainda é fonte de alimento para abelhas [64].

A espécie Tacinga inamoena é uma Cactaceae [65] endêmica do Leste do Brasil [66], com ocorrência em todos os estados da região Nordeste e em Minas Gerais [67, 68]. Esse táxon possui frutos e cladódios que servem de alimento para inúmeros animais [69].

A espécie Turnera subulata, pertencente à família Turneraceae é uma espécie neotropical, encontrada na América Central, Caribe e América do Sul. No Brasil é comum na região Nordeste, florescendo e frutificando durante todo o ano [70]. O táxon fornece pólen e néctar, do qual as abelhas dependem para viver e se produzir [71, 61]. Assim, esse conjunto de informações acerca das espécies nativas excluídas do ambiente invadido evidenciam que os efeitos da exótica invasora vão muito além da redução de números. 
O valor de índice de impacto ambiental obtido no presente trabalho confirma os efeitos danosos da exótica invasora sobre a biota local. Segundo Reaser et al. (2007) [35], valores abaixo de 0,8 representam sérios problemas ambientais para as comunidades autóctones. Em um trabalho realizado na Caatinga com a exótica invasora Prosopis juliflora [50], os resultados foram semelhantes ao presente estudo em que o índice de impacto ambiental foi de -0,635 e -0,637.

Vale ressaltar que o sucesso das invasões biológicas depende da interação entre as adaptações intrínsecas das espécies, das características ecológicas da comunidade, da eficiência dos inimigos naturais, da disponibilidade de recursos adequados e das condições ambientais [72]. As principais teorias sobre as invasões biológicas sugerem que elas sejam conduzidas por flutuações na disponibilidade de recursos, que causam variações na disponibilidade do nicho, independentemente do mecanismo de liberação de recurso [73].

Assim, os resultados obtidos evidenciam os impactos causados pela $B$. diffusa sobre a comunidade autóctone e corrobora com as afirmações de Randall (1996) [74], Primental et al. (2000) [75] e Ziller (2001) [47], que as espécies invasoras geram sérias consequências para os sítios invadidos. Elas alteram a estrutura do habitat, bem como o fluxo de energia e matéria, afetando a composição de espécies da comunidade, riqueza e diversidade $[76,77,45,47,17$, $48,16,49]$.

\section{CONCLUSÃO}

Nossos resultados indicam que a composição, riqueza, abundância de indivíduos, diversidade e estrutura do estrato herbáceo e arbustivo autóctone da Caatinga é fortemente afetado pela invasão da espécie exótica $B$. diffusa. Assim, fica evidente a necessidade de controle dessa espécie exótica invasora, para manutenção da diversidade de espécies da Caatinga em locais invadidos.

\section{REFERÊNCIAS BIBLIOGRÁFICAS}

1. Siqueira Filho JA, Souza DP, Siqueira AA, Meiado MV, Corrêa LC, Campelo MJA, Ramos RRD. A queda do mito: Composição, Riqueza e Conservação das plantas vasculares das Caatingas do Rio São Francisco. Siqueira Filho JA. (Org.) In: Flora das Caatingas do Rio São Francisco: História Natural e conservação. 1. ed. Rio de Janeiro: Andrea Jakobsson Estúdio Editorial; 2012

2. MMA - Ministério do Meio Ambiente. Avaliação e ações prioritárias para a conservação da biodiversidade da Caatinga; 2002

3. Leal IR, Silva JMC, Tabarelli M, Lacher JRTE. Mudando o curso da conservação da biodiversidade na Caatinga do Nordeste do Brasil. Megadiversidade. 2005 Jul;1(1):1-8.

4. Cavalcante A, Major I. Invasion of alien plants in the Caatinga biome. Ambio. 2006 May;35(3):141143, doi:10.1579/0044-7447.

5. Williamson M. Biological Invasions. London: Chapman e Hall; 1996

6. Williamson MH, Fitter A. The characters of successful invaders. Biol Conserv. 1996;78:163-170.

7. Ziller SR. Plantas exóticas invasoras: A ameaça da contaminação biológica. Ciência Hoje. 2001 Dez;30(178):77-79.

8. Fabricante JR, Araújo KCT, Andrade LA, Ferreira JVA. Invasão biológica de Artocarpus heterophyllus Lam. (Moraceae) em um fragmento de Mata Atlântica no Nordeste do Brasil: impactos sobre a fitodiversidade e os solos dos sítios invadidos. Acta Bot Bras. 2012 Fev;26(2):39407.

9. Martins CR, Hay JV, Walter BMT, Proença CEB, Vivaldi LJ. Impacto da invasão e do manejo do capim-gordura (Melinis minutiflora) sobre a riqueza e biomassa da flora nativa do Cerrado sentido restrito. Rev Bras Bot. 2011 Jan;34(1):73-90.

10. Colautti RI, Macisaac HJ. A neutral terminology to define 'invasive' species. Divers Distrib. 2004 Feb;10(1):134-141, doi:10.1111/j.1366-9516.2004.00061.

11. Matthews S. América do sul invadida América do sul invadida. A crescente ameaça das espécies exóticas invasoras. [S.1.]: GISP - Global Invasive Species Programme Secretariat, 2005. 80 p. Disponível em: < http://www.institutohorus.org.br/download/gispSAmericapo.pdf> Acesso em: Maio, 2018.

12. Keane RM, Crawley MJ. Exotic plant invasions and the enemy release hypothesis. Trends Ecol Evolut 2002 Apr;17:164-17, doi:10.1016/S0169-5347(02)02499-0.

13. Baker HG. The evolution of weeds. Annu Rev Ecol Evol Syst. 1974 Nov;5:1-24, doi.org/10.1146/annurev.es.05.110174.000245. 
14. Pegado CMA, Andrade LA, Félix LP, Perreira IM. Efeitos da invasão biológica de algaroba - Prosopis juliflora (Sw.) DC. sobre a composição e a estrutura do estrato arbustivo-arbóreo da caatinga no Município de Monteiro, PB, Brasil. Acta Bot. Bras. 2006 Mai;20(4):887-898.

15. Almeida CFCBR, Amorim ELC, Albuquerque UP, Maia MBS. Medicinal plants popularly used in the Xingo region - a semi-arid location in Northeastern Brazil. J Ethnobiol Ethnomed. 2006 Mar;2(15):19, doi.org/10.1186/1746-4269-2-15

16. Andrade LA, Fabricante JR, Oliveira FX. Impactos da Invasão de Prosopis juliflora (sw.) DC. (Fabaceae) sobre o estrato arbustivo-arbóreo em áreas de Caatinga no Estado da Paraíba, Brasil. Acta Sci Biol Scien. 2010 Abr;32(3):249-255, doi:10.4025/actascibiolsci.v32i3.4535.

17. Andrade LA, Fabricante JR, Alves AS. Algaroba (Prosopis juliflora (Sw.) DC.): Impactos sobre a Fitodiversidade e Estratégias de Colonização em Área Invadida na Paraíba, Brasil. Nat Conserv. 2008 Out;6(2):61-67.

18. Souza VC, Andrade LA, Bezerra FTC, Fabricante J R, Feitosa RC. Avaliação populacional de Sesbania virgata (Cav.) Pers. (Fabaceae Lindl), nas margens do rio Paraíba. Rev Bras Cien Agra. 2011 Abr;6(2):314-320, doi:10.5039/agraria.v6i2a926.

19. Castro R A, Fabricante JR, Araújo KCT. Sociabilidade e potencial alelopático de espécies da Caatinga sobre a invasora Nicotiana glauca Graham (Solanaceae). Natureza online 2016 Out;15(1):59-69.

20. Sousa FQ, Andrade LA, Xavier KRF, Silva PCC, Albuquerque MB. Impactos da invasão por Cryptostegia madagascariensis Bojer ex Decne. (Apocynaceae juss.) em remanescentes de caatinga no município de Ibaretama, Ceará. Ciência Florestal. 2017 Out;27(4):1243-1255, doi: 10.5902/1980509830312.

21. Furtado MDG, Fabricante JR, Siqueira JA. Boerhavia diffusa. In: Fabricante JR. (Org.). Plantas Exóticas e Exóticas Invasoras da Caatinga. Bookess; 2013. 51 p.

22. Dharmaraj G, Babu RC, Natarajaratnam N, Subramani AMS. Allelopathy of certain weed species. Madras Agricult J. 1998;75(3):147-148.

23. Pielou UC. Mathematical diversity. New York: John Wiley; 1997. 385 p.

24. Cavalcanti MAQ, Oliveira LG, Fernandes MJ, Lima DM. Fungos filamentosos isolados do solo em municípios na região Xingó, Brasil. Acta Bot Brasil 2006 Mai;20(4):831-837.

25. Costa RG, Medeiros AN, Alves AR, Medeiros GR. Perspectivas de Utilização da Flor-de-seda (Calotropis procera) na Produção Animal. Rev Caatinga. 2009 Jan;22(1):276-285.

26. Zar JH. Bio estatistical analysis. New Jersey: Prentice Hall; 1999. 639 p.

27. Shannon CE, Weaver W. The Mathematical Theory of Communication. Urbana: University Illinois Press; 1949. 27:617.

28. Pielou UC. Mathematical diversity. New York: John Wiley; 1997. 385 p.

29. Lehmann EL. Testing statistical hypotheses. 2.ed. New York: Springer-Verlag; 1997. 600 p.

30. Menon TA, Carvalho FA. Estrutura populacional de Pinus elliottii em áreas de regeneração florestal em Juiz de Fora, MG. Pesq Florestal Bras. 2012 Dez;32(72):367-372, doi:10.4336/2012.pfb.32.72.367.

31. Brower JE, Zar JH. Field e laboratory methods for general ecology. Duduque, W. C. Brown Publishers; 1984. 226 p.

32. Sokal RR, Rohlf FJ. Biometry: the principles and practice of statistics in biological research. San Francisco; 1981.859 p.

33. Clarke KR. Non-parametric multivariate analysis of changes in community structure. Aust J Ecol. 1993 Mar;18(1):117-143, doi:10.1111/j.1442-9993.1993.tb00438.x

34. Rodal MJN, Nascimento LM. Levantamento florístico da floresta serrana da reserva biológica de Serra Negra, microrregião de Itaparica, Pernambuco, Brasil. Acta Bot Bras. 2002 Mai;16(4):481-500

35. Reaser JK, Meyerson LA, Cronk Q, Poorter M, Eldrege LG, Green E, Kairo M, Latasi P, Mack RN, Mauremootoo J, O'dowd D, Orap AW, Sastroutomo S, Saunders A, Shine C, Thrainsson S, Vaiutu L. Ecological and socioeconomic impacts of invasive alien species in island ecosystems. Environm Cons. 2007 Mai;34(2):98-111, doi:10.1017/S0376892907003815.36.

36. Chaser MW, Christenhusz MJM, Fay MF, Byng JW, Judd WS, Soltes DE, Mabberley DJ, Sennikav AN, Soltis PS, Stevens PF. An update of the Angiosperm Phylogeny Group classification for the orders and families of flowering plants APG IV. Bot J Linn Soc. 2016;181(1), doi:org/10.1111/boj.12385

37. Lista de espécies da flora do Brasil 2018. Disponível em: <http://floradobrasil.jbrj.gov.br/reflora/listaBrasil/PrincipalUC/PrincipalUC.do\#CondicaoTaxonCP> Acesso em: Maio de 2018.

38. Kovach WL. MVSP. A MultiVariate Statistical Package for Windows, ver. 3.1. Pentraeth, Wales, U.K: Kovach Computing Services; 2005.

39. Hammer O, Harper DA, Ryan PD. PAST 1.12. Paleontolological Statistics; 2003.

40. Prach K, Walker LR. Four opportunities for studies of ecological succession. Trends Ecol Evol. 2011 Mar;26(3):119-23, doi:10.1016/j.tree.2010.12.007 
41. Pagad S, Cenoves IP, Carnevali L, Schigel D, Mcgeoch MA. Data Descriptor: Introducing the Global Register of Introduced and Invasive Species. Sientitific Data; 2018.

42. Breytenbach GJ. Impacts of alien organisms on terrestrial communities with emphasis on communities of the south-western Cape. In: Macdonald IAW, Kruger FJ, Ferrara AA. The ecology and management of biological invasions in Southern Africa. Cape Town, Oxford University Press; 1986. p. 229-238.

43. Versfeld DB, Van Wilgen BW. Impact of woody aliens on ecosystem properties. In: Macdonald IAW, Kruger FJ, Ferrara AA. The ecology and management of biological invasions in southern Africa. Cape Town, Oxford University Press; 1986. p. 239-246.

44. Rapoport EH. Contaminação por espécies. Ciência Hoje. 1991;13:52-57.

45. Rejmánek M, Richardson DM, Pysek P. Plant invasions and invasibility of plant communities. In: Van Der Maarel, E. (Ed.), Vegetation Ecology; 2005. p. 332-355.

46. Parker IM, Simberloff D, Lonsdale WM Goodell K, Wonham M, Kareiva PM, Williamson M H, Von Holle B, Moyle PB, Byers JE, Goldwasser L. Impact: Toward a framework for understanding the ecological effects of invaders. Biol Invasions. 1999;1:3-19.

47. Ziller SR, Zalba S. Propostas de ação para prevenção e controle de espécies exóticas invasoras. Natureza \& Conservação. 2007 Out;5(2):8-15.

48. Andrade MVM, Andrade AP, Silva DS, Bruno RLA, Guedes DS. Levantamento florístico e estrutura fitossociológica do estrato herbáceo e subarbustivo em áreas de Caatinga no Cariri Paraibano. Rev Caatinga. 2009 Jan;22(1):229-237.

49. Abreu RCR, Rodrigues PJFP. Exotic tree Artocarpus heterophyllus (Moraceae) invades the Brazilian Atlantic Rainforest. Rodriguésia. 2010; 61(4):677-688, doi:10.1590/2175-7860201061409

50. Fabricante JR, Araújo KCT, Andrade LA, Ferreira JVA. Invasão biológica de Artocarpus heterophyllus Lam. (Moraceae) em um fragmento de Mata Atlântica no Nordeste do Brasil: impactos sobre a fitodiversidade e os solos dos sítios invadidos. Acta Bot Bras. 2012 Fev;26(2):399-407.

51. Andrade LA, Fabricante JR, Oliveira FX. Invasão Biológica por Prosopis juliflora (Sw.) DC: Impactos sobre a Diversidade e a Estrutura do componente arbustivo-arbóreo da Caatinga no Estado do Rio Grande do Norte, Brasil. Acta Bot Bras. 2009;23(4):935-943.

52. Oliveira DG. A família Euphorbiaceae Juss. Em um fragmento de Caatinga em Sergipe. Scientia Plena. 2013 Abr;9(4):1-7.

53. Fabricante JR, Oliveira MNA, Filho JAS. Aspectos da ecologia de Calotropis procera (Apocynaceae) em uma área de Caatinga alterada pelas obras do Projeto de Integração do Rio São Francisco em Mauriti, CE. Rodriguésia. 2013 Jun;64(3):647-654.

54. Sátiro LN, Roque N. A família Euphorbiaceae nas caatingas arenosas do médio rio São Francisco, BA, Brasil. Acta Bot Bras. 2008 Mai;22(1):99-118.

55. Burger W, Huft M. Family 113 Euphorbiaceae. In: Burger W, Huft M. Flora Costaricensis. Fieldiana. 1995;1-169.

56. Melo AL, Sales MF. O gênero Cnidoscolus Pohl (Crotonoideae-Euphorbiaceae) no Estado de Pernambuco, Brasil. Acta Bot Bras. 2008 Jul.;22(3):806-827, doi:10.1590/S010233062008000300017.

57. Melo JG, Araújo TAS, Castro VTNA, Cabral DLV, Rodrigues MD, Nascimento SC, Amorim ELC, Albuquerque UP. Antiproliferative Activity, Antioxidant Capacity and Tannin Content in Plants of Semi-Arid Northeastern Brazil. Molecules. $2010 \quad$ Nov;15(12):8534-8542, doi:10.3390/molecules15128534.

58. Araújo JLO, Quirino ZGM, Neto PCG, Araújo AC. Síndromes de polinização ocorrentes em uma área de Mata Atlântica, Paraíba, Brasil. Biotemas. 2009 Dez;22(4):83-94.

59. Silva CM, Silva CI, Herncir M, Queiroz RT, Imperatriz- Fonseca VL. Guia de plantas visitadas por abelha na Caatinga, 1. Ed. Editora Fundação Brasil Cidadão; 2012. 99 p.

60. Braga LF, Sousa MP, Braga JF, Delachiave MEA. Escarificação ácida, temperatura e luz no processo germinativo de sementes de Senna alata (L.) Roxb. Rev Bras Plantas Med. 2010 Set;12(1):1-7, doi:10.1590/S1516-05722010000100001.

61. Aguiar CML, Santos GMM. Compartilhamento de Recursos Florais por Vespas Sociais (Hymenoptera: Vespidae) e Abelhas (Hymenoptera: Apoidea) em uma Área de Caatinga. Neotr Entomol. 2007 Dez;36(6):836-842

62. Alves FM, Sartori ALB. Caesalpinioideae (Leguminosae) de um remanescente de Chaco em Porto Murtinho, Mato Grosso do Sul, Brasil. Rodriguésia 2009 Abr;60(3):531-550.

63. Machado IC, Sazima M. Pollination and breeding system of Melochia tomentosa L. (Malvaceae), a keystone floral resource in the Brazilian Caatinga. Flora. 2008 Aug;203:484-490, doi:10.1016/j.flora.2007.09.003.

64. Carvalho CAL, Marchini L. Plantas visitadas por Apis mellifera L. no vale do rio Paraguaçu, Município de Castro Alves, Bahia. Rev Bras Bot. 1999 Jun;22(2):333-338. 
65. Menezes MOT, Talor NP, Loiola MIB. Flora do Ceará, Brasil: Cactaceae. Rodriguésia 2013 Jan;64(4):757-774.

66. Hunt D.The new cactus lexicon. Text Volume. DH Books, Milborne Port, 2006. 373 p.

67. Taylor N, Zappi D. Cacti of Eastern Brazil. Royal Botanic Gardens, Kew, 2004. 499 p.

68. Menezes MOT, Taylor N, Machado MC, Coelho PJA, Correia D. Diversity and distribution of Cactaceae in Ceará state, North-eastern Brazil. Bradleya. 2011 Dec;29:13-42, doi:10.25223/brad.n29.2011.a3

69. Sousa AAC, Cordeiro I, Cardiel, JM, Caruzo, MBR. Sinopse do gênero Acalypha L. (Euphorbiaceae) no Estado de São Paulo, Brasil. Hoehnea. 2017 Jul,44(3):336-348, doi:10.1590/2236-8906-79/2016

70. Arbo MM. Estúdios sistemáticos en Turnera (Turneraceae). III Series Anomalae y Turnera. 2005 Jun; 14(3):115-318.

71. Medeiro PCR, Schlindewein, C. Territórios de machos, acasalamento, distribuição e relação com plantas em Protomeliturga turnerae (Ducke, 1907) (Hymenoptera, Andrenidae). Rev Bras Ent. 2003 Out;47(4):589-596.

72. Funk JL, Vitousek, PM. Resource-use efficiency and plant invasion in low-resource systems. Nature. 2007 Apr; 446:1079-1081, doi:10.1038/nature05719

73. Menon TA, Carvalho FA. Estrutura populacional de Pinus elliottii em áreas de regeneração florestal em Juiz de Fora, MG. Pesq Florestal Bras. 2012 Dez;32(72):367-372, doi:10.4336/2012.pfb.32.72.367

74. RandalL JM. Weed control for the preservation of biological diversity. Weed Techn. 1996 Jun;10:370383, doi:10.1017/S0890037X00040124

75. Primental D, Lach L, Zuringa R, Morrison D. Environmental and economic costs on indigenous species in the United states. Bioscience 2000 Jan;50(1):53-65, doi:10.1641/0006-3568(2000)050.

76. Ervin GN, Wetzel RG. Influence of a dominant macrophyte, Juncus effusus, on wetland plant species richness, diversity, and community composition. Oecologia. 2002 Feb;130:626-636, doi:10.1007/s00442-001-0844-x.

77. Eriksson BK, Rubach A, Hillebrand H. Biotic habitat complexity controls species diversity and nutrient effects on net biomass production. Ecology. 2006 Jan;87(1):246-254, doi:10.1890/05-0090 